\title{
Einseitige temporäre Zwerchfellparese nach Bronchialarterienembolisation bei einem Mädchen mit zystischer Fibrose und massiver Hämoptyse: ein Fallbericht
}

\author{
Vito Terlizzi $^{\mathrm{a}}$ Matteo Botti ${ }^{\mathrm{b}}$ Giacomo Gabbani ${ }^{\mathrm{c}}$ Fabrizio Fanelli ${ }^{\mathrm{c}}$ Maurizio de Martino ${ }^{\mathrm{d}}$ \\ Giovanni Taccetti ${ }^{\mathrm{a}}$ \\ ${ }^{a}$ Cystic Fibrosis Centre, Department of Pediatric Medicine, Anna Meyer Children's University Hospital, Florenz, Italien; \\ ${ }^{b}$ Department of Pediatrics, Santa Chiara Hospital, University of Pisa, Pisa, Italien; \\ 'Department of Emergency, Diagnostic and Operative Radiology, Careggi University Hospital, Florenz, Italien; \\ ${ }^{\mathrm{d}}$ Department of Health Sciences and Anna Meyer Children's University Hospital, University of Florence, Florenz, Italien
}

\section{Schlüsselwörter}

Kinder · Zystische Fibrose · Nervus phrenicus · Massive Hämoptyse

\begin{abstract}
Zusammenfassung
Hintergrund: Massive Hämoptyse ist eine schwerwiegende Komplikation bei Zystischer Fibrose (CF), die häufig bei älteren Patienten auftritt. Um die Blutung zu stoppen, kann eine Bronchialarterienembolisation (BAE) durchgeführt werden. Die BAE ist generell ein sicheres und effektives Verfahren, kann jedoch gelegentlich zu schwerwiegenden Komplikationen führen. Wir berichten über den ersten Fall einer temporären einseitigen Zwerchfellparese im Zusammenhang mit einer Lungenkonsolidierung nach BAE bei einem Mädchen mit CF. Durch diese Komplikation verschlechterte sich die Lungenfunktion der Patientin, die sich 9 Monate später einer Lungentransplantation unterzog.
\end{abstract}

Fallvorstellung: Eine 14-jährige Patientin mit CF, die durch das Zentrum für Zystische Fibrose in Florenz beobachtet wurde, zeigte leichtes Fieber, verstärkten Husten und rezidivierende Episoden schwerer Blutungen, so dass man sich zur Durchführung einer BAE entschloss. Innerhalb von 24 Stunden begann die Patientin, über starke Schmerzen in der rechten Thoraxhälfte zu klagen, die mit zunehmender Atemnot und Fieber einhergingen. Eine CT-Angiographie und eine Durchleuchtung ließen die Zwerchfellparese rechts erkennen, die vor dem Eingriff nicht vorgelegen hatte. Nach 4 Tagen hatten sich der klinische Zustand der Patientin sowie der Röntgenbefund verbessert, und die Mobilität der rechten Zwerchfellhälfte war wiederhergestellt. Neun Monate später benötigte das Mädchen eine maschinelle Beatmung, und aufgrund einer pulmonalen Exazerbation mit septischem Schock musste nachfolgend eine extrakorporale Membranoxygenierung (ECMO) eingeleitet werden. Eine Lungentransplantation unter ECMO wurde mit Erfolg durchgeführt.

Schlussfolgerung: Kliniker sollten sich der Möglichkeit einer Verletzung des Nervus phrenicus im Zusammenhang mit einer BAE bei Kindern mit $C F$ bewusst sein.

(C) 2020 The Author(s)

\section{Hintergrund}

Hämoptyse ist eine häufige Komplikation bei Zystischer Fibrose (cystic fibrosis, CF), die bei etwa 9\% der Patienten auftritt [1]. Eine massive oder schwere Hämoptyse wird aktuell definiert als akute Blutung von mehr als $240 \mathrm{ml} /$ Tag oder als rezidivierende Blutungen mit erheblichen Mengen über mehrere Tage [1, 2]. In der pä- diatrischen CF-Population tritt sie bei rund 1-1,5\% der Patienten auf und stellt ein potenziell lebensbedrohliches Ereignis dar [1]. Die perkutane Bronchialarterienembolisation (BAE) stellt eine sichere und wirksame Behandlung bei Patienten mit schwerer Hämoptyse dar [3]. In den Leitlinien der Cystic Fibrosis Foundation wird die BAE bei lebensbedrohlicher Hämoptyse ohne Altersbeschränkungen empfohlen [2]. Nach einer BAE werden häufig
This article is licensed under the Creative Commons Attribution 4.0 International License (CC BY) (http://www.karger.com/Services/Open AccessLicense). Usage, derivative works and distribution are permitted provided that proper credit is given to the au-
Vito Terlizzi
Cystic Fibrosis Centre, Department of Pediatric Medicine

Anna Meyer Children's University Hospital

Viale Gaetano Pieraccini 24, 50139 Florenz, Italien

terlizzivito@libero.it 


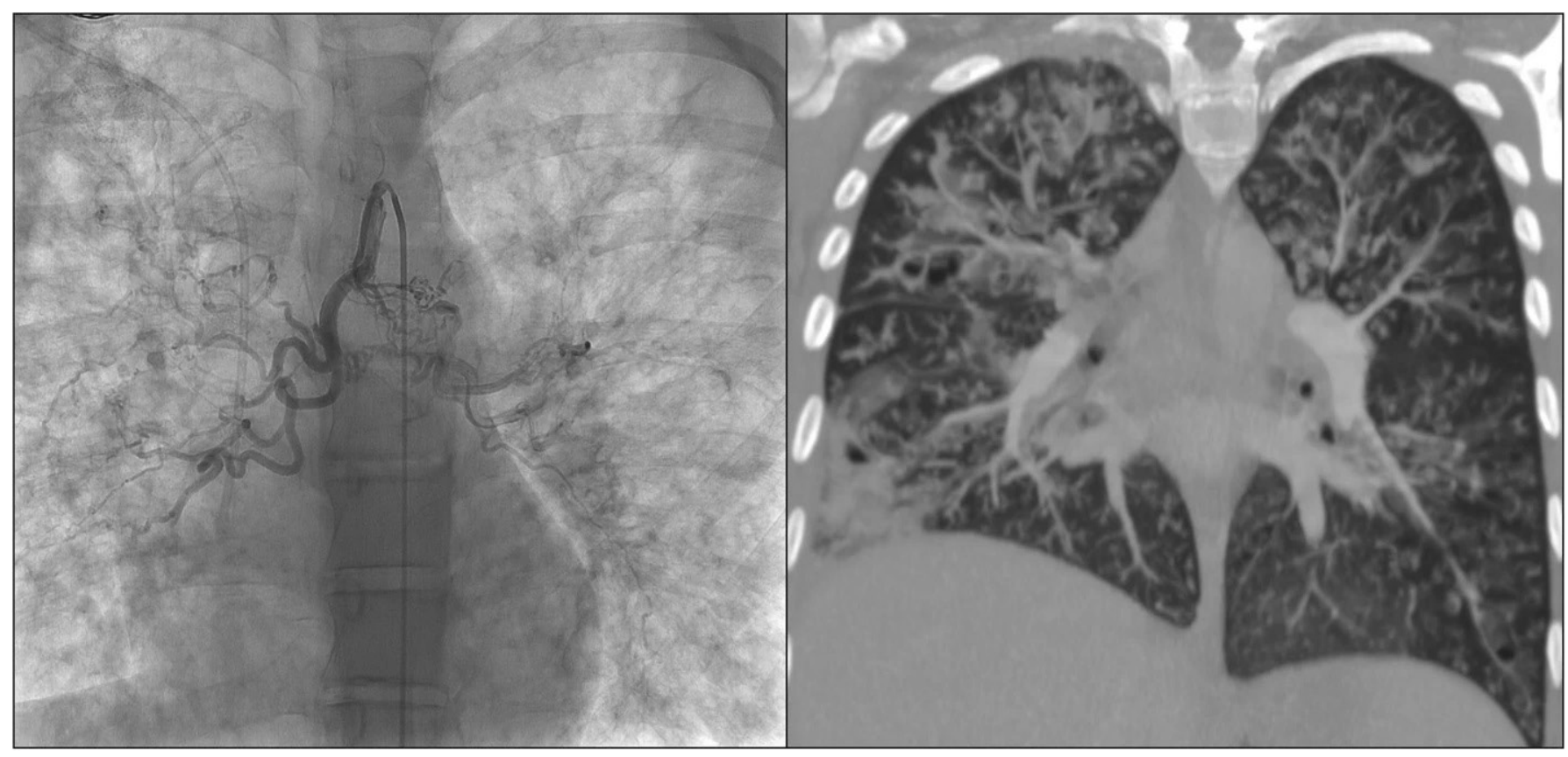

Abb. 1. (a) Angiographie des Hauptastes der Bronchialarterien, (b) Maximumintensitätsprojektion (MIP) coronales Multi-Detektor-Computertomographie (MDCT)-Schnittbild mit Hochstand der rechten Zwerchfellhälfte und gleichzeitiger Lungenkonsolidierung.

Brustschmerzen und Dysphagie berichtet. Schwerwiegende Komplikationen sind selten. Bei einer von diesen handelt es sich um die Embolisation eines Nicht-Zielgefäßes mit nachfolgender Gewebe-Ischämie [3]. Wir stellen einen Fall massiver Hämoptyse bei einem Mädchen vor, der mittels BAE behandelt und durch eine einseitige Zwerchfellparese kompliziert wurde.

\section{Fallvorstellung}

Bei einer CF-Patientin, die durch das Zentrum für Zystische Fibrose in Florenz beobachtet wurde, trat im Alter von 14 Jahren die erste Hämoptyse-Episode auf. In den beiden vorangegangenen Jahren litt sie an chronischer Ateminsuffizienz mit einer Einsekundenkapazität (forced expiratory volume in 1 second, forciertes exspiratorisches Volumen in 1 Sekunde, $\mathrm{FEV}_{1}$ ) von $40-45 \%$ sowie einer chronischen Besiedlung mit Methicillin-resistentem Staphylococcus aureus und einer intermittierenden Besiedlung mit Pseudomonas aeruginosa. Zu Hause traten bei ihr leichtes Fieber, zunehmender Husten und 2 Episoden von Hämoptysen $(<100$ $\mathrm{ml} / \mathrm{Tag}$ ) auf, die initial mit Ruhe, Minocyclin und oral verabreichtem Rifampicin behandelt wurden. In den nachfolgenden Stunden kam es zu weiteren Hämoptyse-Episoden, so dass die Patientin an die pädiatrische Notaufnahme überwiesen wurde. Bei der Aufnahme war sie klinisch stabil. Es wurde eine intravenöse Antibiose (Linezolid und Ceftazidim) eingeleitet. An den folgenden 2 Tagen traten bei dem Mädchen weitere schwere Blutungen (> $200 \mathrm{ml} / \mathrm{Tag}$ ) auf. Eine computertomographische Angiographie (CTA) des Thorax ergab keine aktive Blutung, auch wenn die Bronchialarterien stark geschlängelt und ektatisch waren. Aufgrund des hohen Risikos erneuter Blutungen durch die schwere vaskuläre Lungenerkrankung entschlossen wir uns zur Durchführung einer perkutanen Embolisation. Eine selektive bronchiale Angiographie ergab einen Shunt zwischen der rechten und linken Bronchialarterie. Eine super-selektive Katheterisierung der rechten Bronchialarterie wurde mit Hilfe eines 2,4F-Mikrokatheters erreicht (Abb. 1a). Die Embolisation wurde unter Verwendung sphärischer Mikropartikel (700-900 $\mu \mathrm{m})$ durchgeführt, bis sich im distalen Arteriensegment ein «Stop-flow» zeigte.

Nach der BAE wurden keine weiteren Hämoptyse-Episoden beobachtet. Innerhalb von 24 Stunden begann die Patientin jedoch, über starke Schmerzen in der rechten Thoraxhälfte zu klagen, die mit zunehmender Atemnot und Fieber einher gingen. Aus diesen Gründen wurde eine erneute CTA durchgeführt. Diese zeigte einen Hochstand der rechten Zwerchfellhälfte und ein Konsolidierungsareal im Segmentum basale laterale des rechten Lungenunterlappens bei gleichzeitig bestehendem Pleuraerguss mit vorherrschender Verteilung in der Fissura obliqua (Abb. 1b). Ein Durchleuchtungsbefund bestätigte die Zwerchfellparese rechts, die vor dem Eingriff nicht vorgelegen hatte. Wir stellten die intravenöse Antibiose auf Piperacillin/Tazobactam und Tigecyclin um und verabreichten ein Kortikosteroid i.v. sowie Sauerstoff über eine High-Flow-Nasenkanüle. Nach einer initialen Phase erheblicher Instabilität hatten sich der klinische Zustand der Patientin sowie der Röntgenbefund nach 4 Tagen verbessert, und die Mobilität der rechten Zwerchfellhälfte war wiederhergestellt. Sechs Monate später zeigte die Entwicklung der FEV $\mathrm{F}_{1}$-Werte ein Absinken um 15 Punkte gegenüber dem $\mathrm{FEV}_{1}$ vor der BAE, und die Patientin wurde auf eine Warteliste für eine Lungentransplantation aufgenommen. Neun Monate später benötigte das Mäd- 
chen eine maschinelle Beatmung, und aufgrund einer pulmonalen Exazerbation mit septischem Schock musste nachfolgend eine extrakorporale Membranoxygenierung (extracoporeal membrane oxygenation, ECMO) eingeleitet werden. Eine Lungentransplantation unter ECMO wurde mit Erfolg durchgeführt.

\section{Diskussion und Schlussfolgerungen}

Wir berichten hier über den ersten Fall einer pädiatrischen CFPatientin mit massiver Hämoptyse, bei der nach BAE eine Verletzung des Nervus phrenicus vorlag. Entsprechend beschrieben Chapman et al. einen Fall von einseitiger Zwerchfellparese nach BAE bei einer 29-jährigen Frau infolge einer Embolisation eines Nicht-Zielgefäßes als unbeabsichtigten Verschluss der Arteria pericardiacophrenica [4]. In unserem Fall ergab die Angiographie weder eine Verbindung der rechten Bronchialarterie zu anderen Gefäßen (Brustwandarterie, Zwerchfellarterie), noch eine Verbindung zum Innervationsgebiet des Nervus phrenicus (Abb. 1a). Wir empfehlen jedoch nachdrücklich, bei Patienten differenzialdiagnostisch die Möglichkeit einer akuten Lungenembolie zu berücksichtigen.

Funktionsstörungen des Zwerchfells sind in der Regel auf eine Dysfunktion des Nervus phrenicus zurückzuführen, die verschiedene Ursachen haben kann: maligne Infiltration, eine Verletzung des Nervs während eines thoraxchirurgischen Eingriffs sowie Erkrankungen des peripheren oder zentralen Nervensystems [5]. In zahlreichen Fällen wird eine isolierte Zwerchfellneuropathie unklarer Genese als idiopathisch klassifiziert. Als zugrunde liegende Ursache werden hierbei Infektionsprozesse vermutet [5].

Möglicherweise sind Funktionsstörungen des Zwerchfells unterdiagnostiziert. Dabei können sie sich negativ auf die Lebensqua- lität auswirken, Hinweise auf den Schweregrad einer Erkrankung geben und - in manchen Fällen, wie etwa auf der Intensivstation - von prognostischem Wert sein.

Die BAE ist bei massiver Hämoptyse zweifellos ein lebensrettender Eingriff, kann jedoch auch bei Patienten im Kindesalter zu schweren Komplikationen führen. Das Risiko-Nutzen-Verhältnis ist in jedem Fall sorgfältig abzuwägen. Insbesondere eine terminale CF kann einen wichtigen Risikofaktor darstellen. Kliniker sollten sich der Möglichkeit einer Verletzung des Nervus phrenicus bei einer BAE bewusst sein und den Patienten nach dem Eingriff auf Brustschmerzen und Dyspnoe überwachen, da diese Komplikation unvorhersehbar ist und keine speziellen Maßnahmen zur Vorbeugung bekannt sind.

\section{Disclosure Statement}

Die Autoren erklären, dass keine Interessenskonflikte bestehen.

\section{Lizenzangabe}

Terlizzi V, Botti M, Gabbani G, Fanellic F, de Martinod M, Taccetti G: Unilateral temporary diaphragmatic paralysis secondary to bronchial artery embolization in a girl with cystic fibrosis and massive hemoptysis: a case report. BMC Pulm Med. 2020;20(1):38. (DOI: 10.1186/s12890-020-10763). ${ }^{\circ} 2020$ The Author(s). (Übersetzung; Verfügbarkeit der Daten und Materialien, Beiträge der Autoren, Danksagungen, Finanzielle Unterstützungen, Ethikerklärungen, Abkürzungen und Publisher's Note gekürzt), lizensiert unter CC BY 4.0 (https://creativecommons.org/licenses/by/4.0/deed. de).Rechte und Berechtigungen

\section{Literatur}

Die Literatur ist unter www.karger.com/Article/Fulltext/507907 abrufbar. 